\title{
A synchronous motor rotor initial position detection methods
}

\section{Chen Zhengshi ${ }^{1}$,Liao Xiaowen ${ }^{2}$ 'Liu mei ${ }^{2}$}

1. Guangzhou vocational college of science and technology, Guangzhou, 510550, China, 2.Guangdong Institute of Petrol-Chemical Technology, Maoming Guangdong,525000, China;

\author{
E-mail: czsls@163.com
}

Key words: IPMSM; initial position ; rotor poles; tracking differentiator

\begin{abstract}
Sources of error are took into account while applying high frequency rotation voltage injection method to interior permanent magnet synchronous motor (IPMSM). A new method using the phase difference of two new tracking differentiator (NTD) output signals for solving the initial position of the rotor poles on the basis of the derivation of high frequency positive, negative sequence current is proposed. Method only need four parameters: bus voltage, electrical angle of high-frequency signal and the inductance of direct and quadric axis, without using PI regulator or Luneburg observer. The experimental results verify the accuracy of the detection. The method can be used to solve the starting problem of the IPMS
\end{abstract}

\section{Introduction}

Interior permanent magnet synchronous motor (IPMSM) rotor pole position is very important for the initial start time of vector control decoupling. High ${ }^{[]}$frequency signal injection method is the initial position detection method without the mainstream position sensor, including highfrequency rotation voltage, high-frequency ripple voltage signal injection method two. High frequency voltage signal injection method rotation detected current signal is converted to a highfrequency carrier voltage reference coordinate system synchronized, filtered positive sequence and fundamental component to obtain the error signal and then using heterodyne processing method as Rhomberg observer or phase-locked loop input, and then estimate the initial position of the rotor poles. However, this method fundamental current regulator and the DC link harmonic position estimation results a great impact ${ }^{[}$[3-5]. Dither voltage signal injection method is based on highfrequency signal injection frequency impedance maximum At the time of $0^{\circ}, 180^{\circ}$, and minimum injection frequency impedance principle detect the initial position of the rotor poles. However, the method by the non-ideal characteristics of the motor structure influenced ${ }^{[]}[6-8]$. Given the strong tracking differentiator tracking capabilities, can extract weak signals buried in the noise, but the functional form of the complex ${ }^{[]}[9]$. The author proposes in the previous study, a novel tracking differentiator(NTD), while maintaining the disturbance rejection and signal tracking capability, greatly simplifying the form ${ }^{\left[{ }^{3}\right.}[10]$. The NTD article applies to negative sequence component rotating high frequency voltage injection method of treatment, to explore a new method IPMSM initial position detection. Method only with bus voltage, high frequency signal electrical angle and cross straight axis inductance parameters without PI regulator or Rhomberg observer, simple and convenient. 


\section{PMSM Rotor Position Detection Method}

Figure 1 is a block diagram of detection of the initial position of the rotor IPMSM proposed. The figure produced by the high-frequency SVPWM algorithm rotation signal injection interior permanent magnet synchronous motor (IPMSM). Let the high-frequency signal amplitude is $u_{s}$ the electrical angle is $\theta_{e}$, axis voltage is $u_{s \alpha} 、 u_{s \beta}$ for two-phase stationary coordinate,motor A、B phase current is $i_{a} 、 i_{b}$, two-phase stationary coordinate current through CLARK transform is $i_{s \alpha} i_{s \beta}$. To use $i_{s \alpha}, i_{s \beta}, \theta_{e}$ and new tracking differentiator NTD1, NTD2 available initial magnetic pole position of the rotor angle $\theta_{r}$. Below is the test of mathematical formula is derived in detail. The definition of a high-frequency signal with respect to the resultant vector $\mathbf{v}$ of instantaneous two-phase stationary coordinate $\alpha$ axis angle is $\theta_{e}$, the initial position of the rotor magnetic pole direction of $\mathrm{d}$ axis, $\mathrm{Q}$ axis and $\mathrm{d}$ axis for the quadrature axis, The $\mathrm{d}$ axis relative to the $a$ axis Angle is $\theta_{r}, \theta_{e} \theta_{r}$

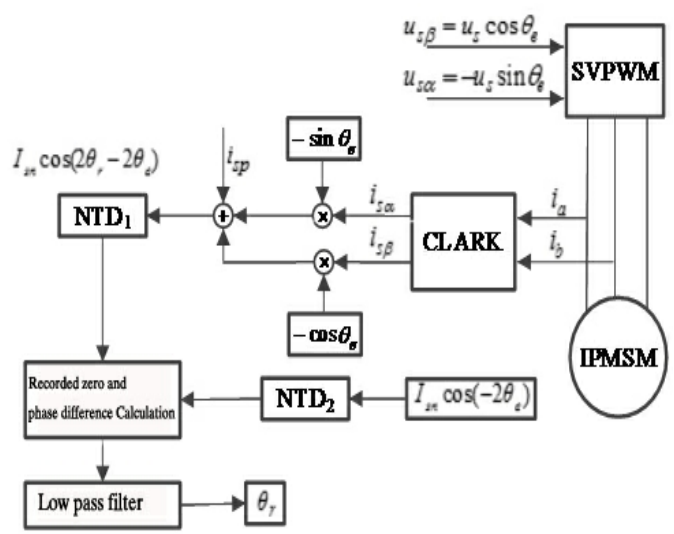
Angle diagram in figure 2 ,In the two-phase stationary coordinates into high-frequency voltage signal amplitude $u_{s}$, angular frequency $\omega$ (as shown in figure 1),the stator flux is formed as follows:

$$
\lambda=\frac{u_{s}}{\omega}\left[\begin{array}{c}
\cos \theta_{e} \\
-\sin \theta_{e}
\end{array}\right]
$$

\section{Figure 1 IPMSM rotor initial position detection principle block diagram}

So that d, q-axis inductance for $L_{d^{\prime}} L_{q}, \quad L=\left(L_{d}+L_{q}\right) / 2, \quad \Delta L=\left(L_{q}-L_{d}\right) / 2$. By PARK inverse transformation and intersecting axis mutual inductance principle [11], two phase static inductance matrix of coordinates are as follows:

$$
\left[L_{s \alpha}, L_{s \beta}\right]=\left[\begin{array}{cc}
L-\Delta L \cos 2 \theta_{e} & -\Delta L \sin 2 \theta_{e} \\
-\Delta L \sin 2 \theta_{e} & L+\Delta L \cos 2 \theta_{e}
\end{array}\right]
$$

If the input signal amplitude is small, the rotor flux to the

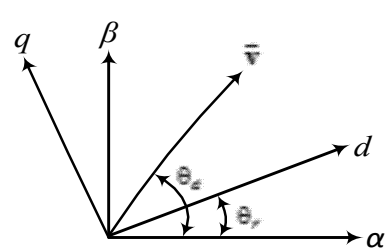

Figure $2 \theta_{r}, \theta_{e}$ Angle diagram

$$
\begin{aligned}
& {\left[\begin{array}{c}
i_{s \alpha} \\
i_{s \beta}
\end{array}\right]=\left[\begin{array}{c}
I_{s p} \sin \theta_{e}-I_{s n} \sin \left(2 \theta_{r}-\theta_{e}\right) \\
I_{s p} \cos \theta_{e}-I_{s n} \cos \left(2 \theta_{r}-\theta_{e}\right)
\end{array}\right]} \\
& \Rightarrow I_{s n} \cos \left(2 \theta_{r}-2 \theta_{e}\right)=-i_{s \alpha} \sin \theta_{e}-i_{s \beta} \cos \theta_{e}+I_{s p}
\end{aligned}
$$

stator flux has little effect, then there is:

$$
\boldsymbol{\lambda}=\left[L_{s \alpha}, L_{s \beta}\right]\left[\begin{array}{l}
i_{s \alpha} \\
i_{s \beta}
\end{array}\right]
$$

So that high frequency positive, negativesequence current magnitude respectively for: $I_{s p}=\frac{U_{s} L}{\omega\left(L^{2}-\Delta L^{2}\right)}, \quad I_{s n}=\frac{U_{s} \Delta L}{\omega\left(L^{2}-\Delta L^{2}\right)}$, by the formula (1) , (3) available 
Formula left corner $I_{s n} \cos \left(2 \theta_{r}-2 \theta_{e}\right)$ contains the initial position $\theta_{r}$ of the rotor poles, and $\theta_{e}=\omega t$ for known quantity. But the method of using $\left(-i_{s \alpha} \sin \theta_{e}-i_{s \beta} \cos \theta_{e}+I_{s p}\right)$ formula estimating $\theta_{r}$ directly, the test results will be affected by current measurements, the bus voltage fluctuation and intersecting axis inductance parameter error factors. The following method to discuss the application of double NTD.

The $Z=\left[z_{1}(t), z_{2}(t)\right]^{T} \in \mathbf{R}^{2}$ for system state variables of $\Sigma, t \in[0, T]$, input signal $u(t)=-i_{s \alpha} \sin \theta_{e}-i_{s \beta} \cos \theta_{e}+I_{s p}$, the valid signal tracking value $z_{1}(t)$ and differential signals $z_{2}(t)$, can get NTD1 as follows:

$$
\begin{aligned}
\delta(t)= & z_{2}(t) \\
& -R^{2 \beta} a\left[\left(z_{1}(t)-u(t)\right) s+\left(z_{1}(t)-u(t)\right)^{\alpha}(1-s)\right] \\
& -R^{\beta} b \mathrm{z}_{2}(h)
\end{aligned}
$$

Where: $s=\frac{\operatorname{sign}\left(z_{1}(t)-u(t)+\delta\right)-\operatorname{sign}\left(z_{1}(t)-u(t)-\delta\right)}{2}, \delta$ is a positive number and tracking accuracy associated results; and $\alpha=\frac{p-1}{p+1}, \mathrm{P}$ is an even number greater than or equal to $2, R^{2 \beta}$ for tracking factor, decided to track speed; a,b is positive number, if a fixed, $\mathrm{b}$ larger system damping $R^{\beta} b$ more Great, reasonable b, to avoid high-frequency vibrations into the steady state without overshoot. To meet the Lyapunov asymptotic stability conditions $\left|\forall\left(z_{1}(t)-u(t)\right)\right| \leq(a / b)^{(p+1) / 2}$. the formula $I_{s n} \cos \left(2 \theta_{r}-2 \theta_{e}\right)$ is obtained by formula (5) the presence of the processing delay, which is hereby incorporated NTD2 process $I_{s n} \cos \left(-2 \theta_{e}\right)$ another way of the same parameters, to offset the impact of the delay on the test results, and then calculate the phase difference obtained $\theta_{r}$.

\section{Experimental Research}

Hiromi experiment was produced by SM8013 type IPMSM, phase resistance $R=1.858 \Omega$, d-axis inductance $L_{d}=6 \mathrm{mH}$, q-axis inductance $L_{q}=13 \mathrm{mH}$, rotor pole pairs $\mathrm{P}=4$ Rotating high frequency voltage amplitude $U_{s}=60 \mathrm{~V}$, frequency $\omega=500 \times 2 \pi \mathrm{rad} / \mathrm{s}$, current sampling interval $T=0.05 \mathrm{~ms}$.

NTD1 experimental parameters used are: $R=100, a=24, \quad b=18, \quad \alpha=0.6, \quad \beta=1, \quad \delta=0.1$.NTD2 process $I_{s n} \cos \left(-2 \theta_{e}\right)$ using the same parameters obtained NTD1 have the same phase lag signal. Adjusting the rotor poles to $\pi / 3, \pi / 4, \pi / 6$ everywhere encoder pulse signal according to the manual. Figure 3-6 horizontal axis represents time and the ordinate the use of a normalized value represents the current size. Figure 3 is $i_{s \alpha}$ current waveform verification method for the anti-disturbance capacity, for a given voltage amplitude $u_{s}$ superimposed Gaussian noise to simulate the bus harmonic current sampling interference.Figure $4 \sim 6$ respectively using NTD1, NTD2 processed signal, post-processing $-i_{s \alpha} \sin \theta_{e}-i_{s \beta} \cos \theta_{e}+I_{s p}$ by NTD1, to clear $I_{s n} \cos \left(2 \theta_{r}-2 \theta_{e}\right)$. Figure 7 is based on the zero crossings and butterworth lowpass filter obtained $\theta_{r}, \theta_{r}$ can converge in the vicinity of the true value of $4 \mathrm{~ms}$, the maximum estimated error 0.082rad. 


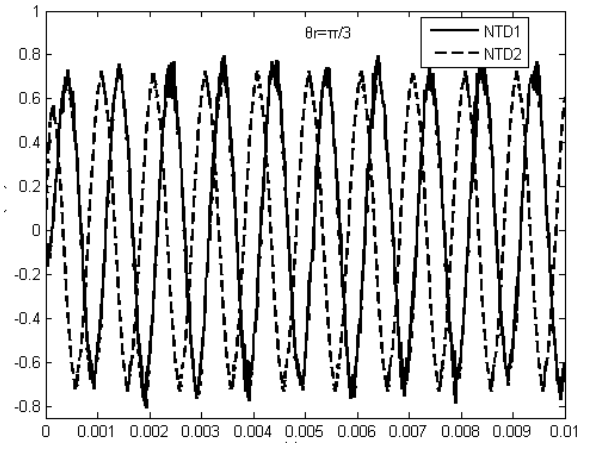

Figure 3 two-phase static $i_{s \alpha}$ current waveform

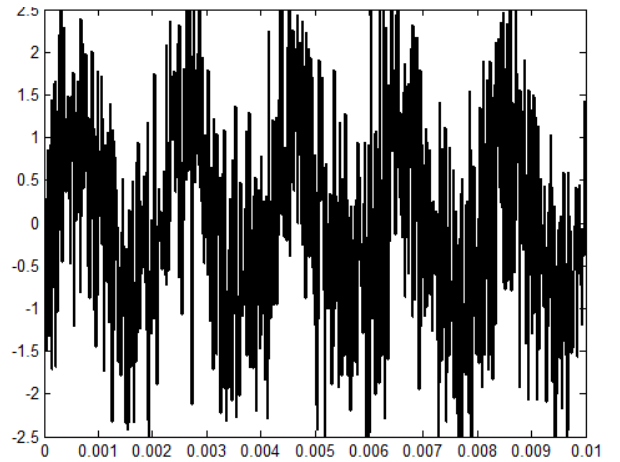

Figure $5 \theta_{r}=\pi / 4$ NTD1, NTD2 output curve

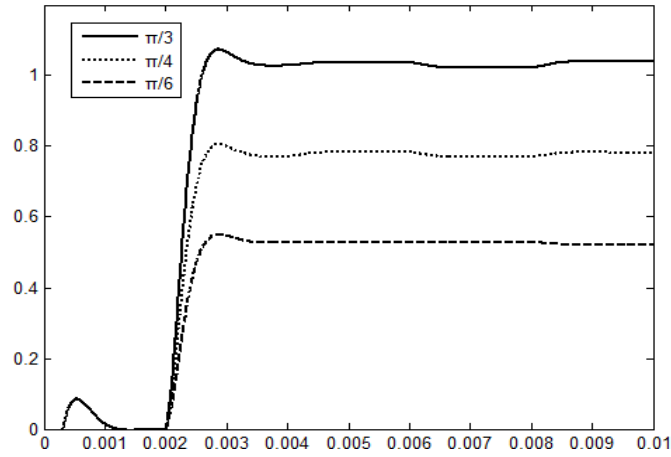

Figure 7 to join after low pass filtering curve $\theta_{r}$

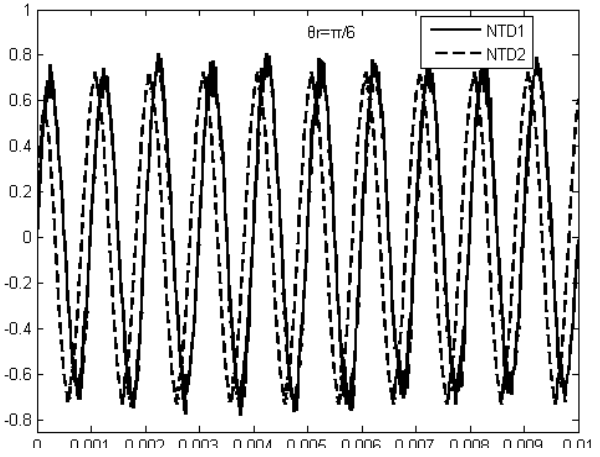

Figure $4 \theta_{r}=\pi / 3$ NTD1, NTD2 output curve

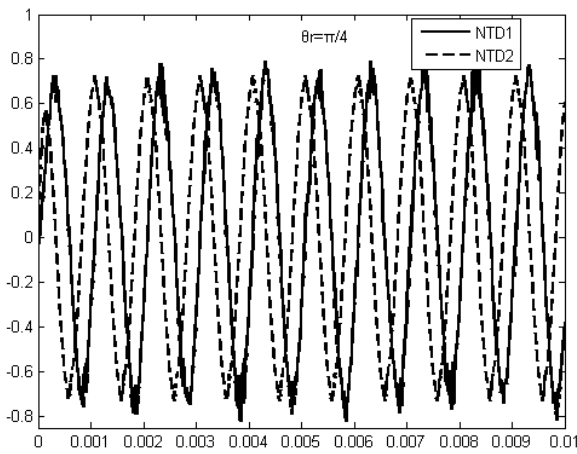

Figure6 $\theta_{r}=\pi / 6$ NTD1, NTD2 output curve

\section{Conclusions}

The accuracy of the initial position detection of rotor poles had a great influence on startup performance.Based on heterodyne method was designed using a two way NTD output signal of the phase difference method to solve the initial position of rotor pole.The results showed that:

(1) method is not sensitive to disturbance, the true value $I_{s n} \cos \left(2 \theta_{r}-2 \theta_{e}\right)$ of NTD can be quickly acquired; (2)the initial position of the rotor poles are slight fluctuations, but does not affect the startup performance; (3relatively heterodyne method, without high frequency positive sequence current filtering, and save the Rhomberg observer and part of the PI regulator, is a heterodyne method improvement. In addition, the physical meaning of NTD clear parameters, parameter adjustment heterodyne relatively easy; (4)method is easy to implement dynamic detection of the rotor magnetic pole position, but the lag filter is large, the future will explore this aspect IPMSM NTD running at low speed without position sensor application problems . 


\section{Reference}

[1] Chen initially positioned based on incremental photoelectric encoder disk PMSM rotor position [J] Motor \& Control Application, 2007,34 (3): 33-36.

[2] Yu-Seok Jeong, Lorenz RD, Seung-Ki Sul. Initial rotor position estimation of an interior permanent-magnet synchronous machine using carrier-frequency injection methods [J]. IEEE Trans. Ind. Application, 2005,44 (1 ): 38-45.

[3] GD Andreescu, CI Pitic, F Blaabjer.Combined flux observer with signal injection enhancement for wide speed range sensorless direct torque control of IPMSM drives [J], IEEE Transactions on Energy Conversion, 2008,23 (2): 393-402 .

[4] Antti Piippo, Marko Hinkkanen, Jorma Luomi.Analysis of an Adaptive Observer for Sensorless Control of Interior Permanent Magnet Synchronous Motors [J] .IEEE TRANS-ACTIONS ON INDUSTRIAL ELECTRONICS [J], 2010,55 (2): 570- 576.

[5] Zhu Xiaohui, Li Yinghui, Chenya Bin no position sensorless vector control based on nonlinear state observer of PMSM [J] Electrical Technology, 2010,25 (1): 50-57.

[6] PROCEEDINGS PROCEEDINGS detect the initial position of the permanent study of highfrequency synchronous motor rotor injection method [J]. Chinese CSEE, 2007,23 (15): 15-20.

[7] Wang Gaolin, Yangrong Feng, Li Gang-based IPMSM no position sensor frequency signal injection control strategy [J] Electrical Technology, 2012,27 (11): 62-68.

[8] to Yan Jun, Chai Feng, Europe King and other high-frequency signal control method based on the rotation position sensor IPMSM [J] Electrical Technology, 2013,28 (7): 62-68.

[9] Han Jingqing, Yuan Lulin tracking differentiator discrete form [J] Systems Science and Mathematics, 1999,19 (3): 268-273.

[10] Liao Xiaowen, Liu Mei detection [J] based on the new tracking differentiator brushless DC motor commutation position Chinese test, 2013,39 (6): 99-104.

[11] Huang Lei, Guang Zhou, Heng based on estimates of the potential expansion of the antiinterpolating sensorless control of permanent magnet synchronous motor [J] Chinese CSEE, 2007,09: 59-63. 